Néanmoins il convient d'insister sur ce fait que, dans quelques cas, la méthode Gerber-Roeder peut fournir un chiffre différant de celui de la méthode au sable par 1 gramme en plus ou en moins \% de fromage frais, c'est-à-dire approximativement $2 \%$ sur sec.

La prudence conseille donc, dans l'utilisation de la méthode Roeder seule, de ne considérer le fromage comme légal que si cette technique le classe comme titrant $2 \%$ sur sec de plus que le chiffre limite. C'est là, à notre avis, une marge de sécurité nécessaire et suffisante.

Si la méthode Gerber donne un chiffre compris à l'intérieur de cette marge (par exemple : 41 ou a fortiori 40 pour un fromage devant titrer $40 \%$ ), il convient de refaire l'analyse par la méthode au sable afin de connaître la teneur exacte en matière grasse.

$$
*^{*} *
$$

\title{
IV. CONCLUSIONS.
}

1. Dans les mises au point de fabrication, dans les cas douteux ou limites, dans les contrôles irréguliers ou rares, il faut appliquer, comme procédé industriel de dosage de la matière grasse des fr6mages, la méthode dite "au sable" en suivant scrupuleusement les recommandations que nous avons exposées plus haut.

2. Dans le contrôle quotidien d'une fabrication déjà bien au point (contrôle de surveillance en grande série) on peut appliquer la méthode Gerber-Roeder en tenant compte de ce fait que, sur le produit frais elle peut laisser subsister, dans quelques cas, une erreur maxima de \pm 1 gramme (c'est-à-dire pratiquement $\pm 2 \%$ sur sec dans la majorité des fromages).

Dès que cette méthode indique que le fromage contient moins de $2 \%$ de matière grasse sur sec au-dessus du chiffre légal, il faut revenir à la méthode au sable pour savoir si cette marge atténuée existe encore réellement.

Un laboratoire industriel de fromagerie, qui exécute des contrôles quotidiens, doit être équipé pour appliquer concurremment ces deux méthodes simples et d'emploi facile.

\section{ACTINISATION DU LAIT}

$\begin{array}{ccc}\text { JEAN VIEILLY } & \text { par } \\ \begin{array}{c}\text { Docteur-Vétérinaire, Industriel laitier, } \\ \text { Grenoble. }\end{array} & \text { et } & \text { JEAN HARDER } \\ \text { Ingénieur Agronome E. P. Z., }\end{array}$

Dans la gamme des radiations dont les longueurs d'ondes vont du millionième de $\mu$ à $30 \mathrm{~km}$., les rayons ultra-violets occupent une place moyenne avec 100-4.000 Angstroms, e'est-à-dire $1 / 100^{\circ}$ 
à $4 / 10^{\mathrm{e}}$ de $\mu$. Ils sont situés entre les rayons $\mathrm{X}$ et les rayons lumineux. Sur les limites, leurs propriétés tendent vers celles des voisins, mais. ils possèdent en propre des propriétés chimiques. En photographie, ce sont eux qui impressionnent les pellicules.

En biologie, on leur a reconnu des propriétés microbicides et une action très importante sur la vitamine $D$.

Mais l'irradiation ne peut se comprendre que pour la surface des corps opaques, solides ou liquides, et pour les masses transparentes aux rayons ultra-violets. Pour ces derniers, l'action chimiquea lieu progressivement; par exemple, il faut une demi-heure d'irradiation pour traverser et impressionner dans toute son épaisseur 4/10 $10^{\mathrm{e}}$ de millimètres de gélatine avec une intensité donnée : 1 seule lampe de quartz au mercure placée à 1 mètre de distance.

Avec le lait, liquide opaque, des essais nombreux ont été tentés. On a cherché à obtenir une espèce de pasteurisation ou stérilisation du lait tout en augmentant la teneur en vitamine $D$.

En France, peu d'essais à notre connaissance ont été tentés parce que en irradiant le lait en couches minces, à l'air libre on obtenait des laits ozonisés, à goût d'huile de foie de morue peu appréoié des. consommateurs, et si l'irradiation se fait sous gaz neutre (l'acide carbonique par exemple), l'installation est d'un prix coûteux et l'exploitation peu commode. Il est difficile, d'autre part, que le lait traité de cette manière ne présente pas un léger goût d'ozone.

A l'étranger, des essais plus nombreux ont été faits. On vend encore en Suisse du lait «Guigoz " desséché, irradié, qui n’a pas eu en France la vogue du simple lait "Guigoz» sec.

En Allemagne, du lait irradié se boit, paraît-il, à Francfort. En Amérique et en France "Dryco " fabrique du lait sec irradié.

Ainsi done jusqu'à ce jour, malgré les propriétés remarquables des rayons ultra-violets, leur application en laiterie a fait peu d'adeptes.

Un inventeur grenoblois, M. W. P. de Stoutz, vient de construire un appareil à irradier les liquides en couches minces dans des. tubes capillaires en quartz. Il a bien voulu nous demander d'expérimenter industriellement son appareil sur le lait, transformant ainsi ma laiterie en un laboratoire d'essais.

Par analogie avec l'actino-thérapie, nous avons donné le nom d" "actinisation" du lait à l'opération qui consiste à faire agir les rayons ultra-violets sur le lait aux fins de l'obtenir :

$1^{0}$ Plus hygiénique, par la disparition de la plus grande quantité des microbes dangereux et la conservation des bacilles. lactiques.

$2^{\circ}$ Plus nourrissant, par l'augmentation de la vitamine D. 
L'actinisation ne doit donc pas donner un lait transformé comme dans la pasteurisation, mais un lait amélioré par la sélection microbienne et par l'activation des vitamines.

Pour l'actinisation comme pour la pasteurisation il ne faut pas croire que l'on pourra rendre bons les laits mauvais. Ils deviendront moins dangereux, les microbes seront détruits, mais il reste leurs toxines. C'est pour cela qu'avec ces deux méthodes, nous considérons qu'il ne faut employer que des laits normaux, remplissant toutes les conditions prévues par les considérants, décrets et circulaires de la loi du 2 juillet 1935 .

Les rayons ultra-violets remplaçant la chaleur, on traite le lait à froid, en ne modifiant pas sa composition chimique, albumine non coagulée, $\mathrm{CO}^{2}$ respecté,

Pour l'actinisation comme pour la pasteurisation, il faut d'abord une sélection, puis un pré-filtrage, et du froid en dernier lieu.

L'actinisation doit donner au lait d'hiver, lait pauvre en vitamine D, par suite de la stabulation dans les étables des vaches nourries au fourrage sec, les qualités du lait du printemps en montagne, du soleil et des fleurs, en un mot le lait véritable "source de vie $)$.

De même qu'il y a une technique pour l'actino-thérapie, il y a une technique de l'actinisation. C'est cette technique que nous exposerons plus tard. D'ores et déjà nous pouvons donner certaines affirmations qui sont de nature à nous faire persévérer dans les recherches techniques et mêmes scientifiques.

\section{CONSIDÉRATIONS INDUSTRIELLES}

\section{Appareil W. P. de Stoutz (Actinisateur W. P. de Stoutz).}

L'invention de M. de Stoutz (brevets demandés dans tous les pays) a pour objet un appareil destiné au traitement des liquides ou gaz en vue de leur activation par irradiation, leur chauffage, refroidissement, en couches très minces, et à l'abri des émanations d'ozone, dans des tubes capillaires perméables aux rayons ultraviolets de certaines longueurs d'ondes.

Il a construit un appareil d'une très belle présentation et d'un encombrement très réduit, un peu plus d'un demi-mètre cube, pour un débit horaire de 1.500 litres de lait ou 1.800 litres d'eau sous pression de $1 / 10^{e}$ d'atmosphère. Un dispositif spécial lui permet d'obtenir très simplement une variation des intensités d'irradiation allant de 1 à 1.000. Toutes les pièces de l'appareil sont facilement échangeables et parfaitement standard. Une prise de terre soigneusement établie évite tout danger et l'ensemble de l'appareil constitue une cage de Faraday parfaite, évitant ainsi tous les parasites pour les amateurs de T. S. F. voisins. J'ajouterai que les portes d'accès 
sont parfaitement étanches comme du reste l'ensemble de l'appareil, ce qui évite des dégagements d'ozone au moment de l'opération et en limite la production à la quantité d'oxygène contenue à l'intérieur de l'appareil. Le nettoyage même de l'appareil se fait de l'extérieur,

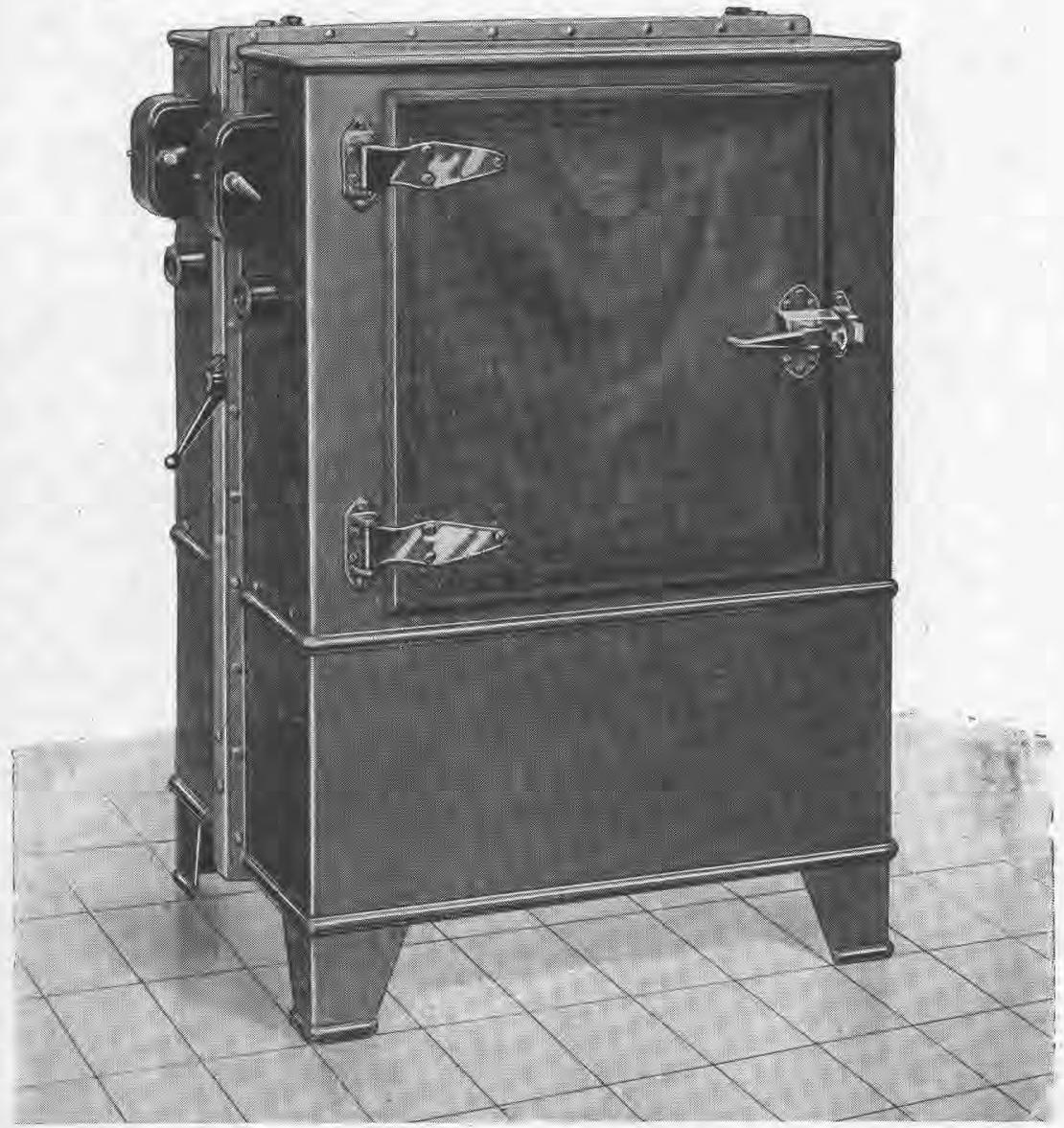

Actinisateur : débit 1.800 litres heure (Vue de la Commande électrique.)

sans qu'il soit besoin d'ouvrir les portes. Un petit regard permet de se rendre compte sans danger de ce qui se passe à l'intérieur.

Un grand nombre de tubes capillaires forment une espèce de harpe lumineuse, dans les cordes de laquelle s'étireraient les gouttes de lait. La surface toujours changeante de celles-ci est d'une grande mobilité puisque la goutte met un dixième de seconde pour traverser les tubes, permettant d'irradier chaque partie de la masse en un temps voisin de un millième de seconde. La couche à irradier est 
alors d'une épaisseur de 1/100 $\mathrm{mm}$. C'est réellement la couche mince. Pendant l'opération, il se produit une légère augmentation de la température du lait qui est de l'ordre de $2 / 10^{\circ}$ de degré et qui par conséquent ne gêne en rien l'irradiation.

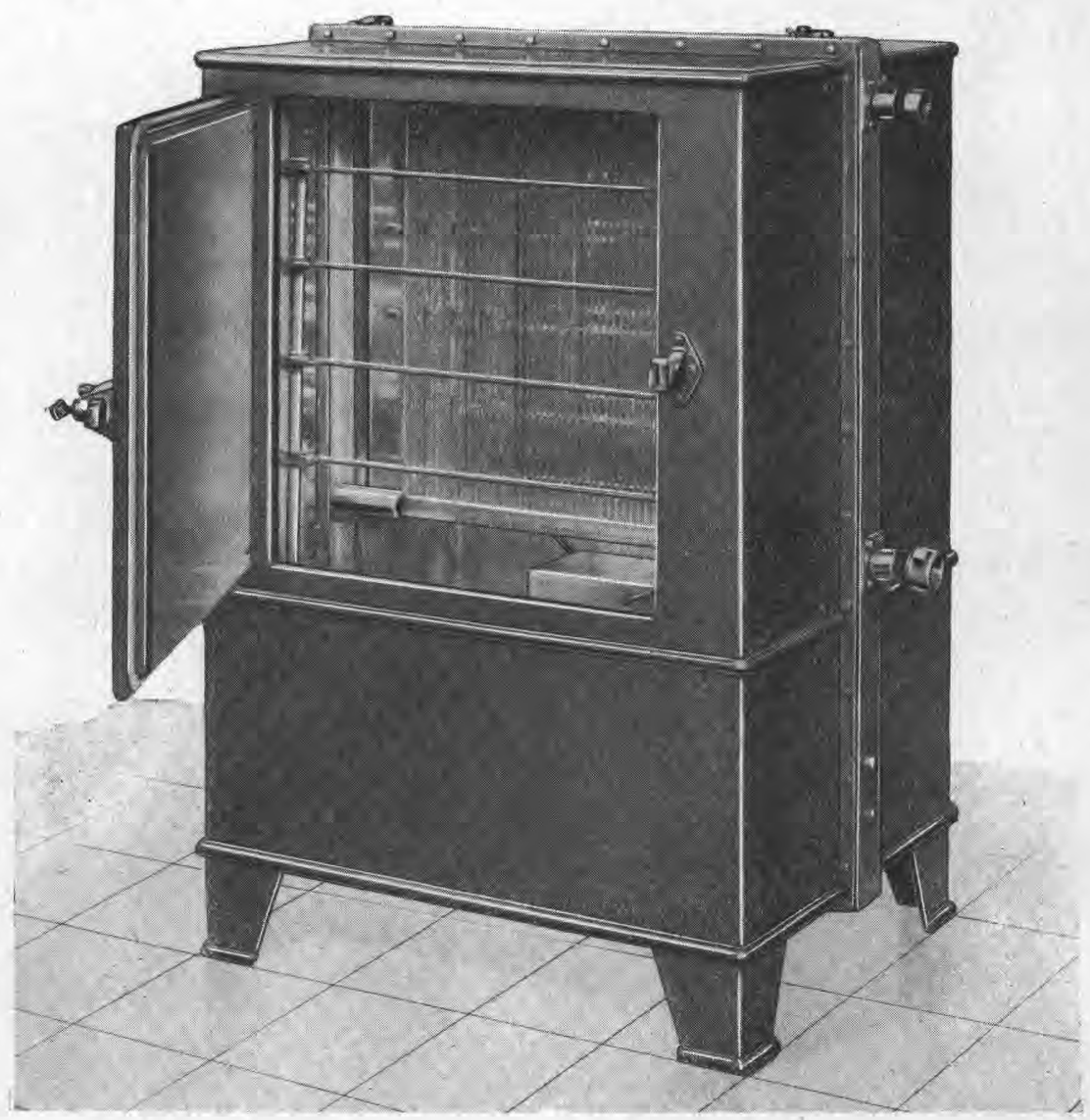

Actinisateur : débit 1.800 litres, ouvert.

(Montrant la disposition de la Herse lumineuse.)

Le cheminement dans les tubes capillaires d'une goutte de lait présente un régime parfait d'écoulement laminaire. Au sujet de cet écoulement je me permets de citer CRUSSARD :

"Le régime laminaire paraît avoir été étudié pour la première fois par PoISEUIL pour les tubes capillaires ; le but de ces essais était la recherche des lois de la circulation du sang. Il a été longtemps considéré par les techniciens comme une curiosité sans intérêt pratique. Et cependant, dans les fuites et les filtrations, les énormes 
écarts auxquels donne lieu l'application des formules usuelles de résistance tient, à n'en pas douter, à un régime laminaire que ces formules ignorent " (1).

M. W. P. de Stoutz, reprenant ces études, a appliqué pour $1_{\mathrm{a}}$

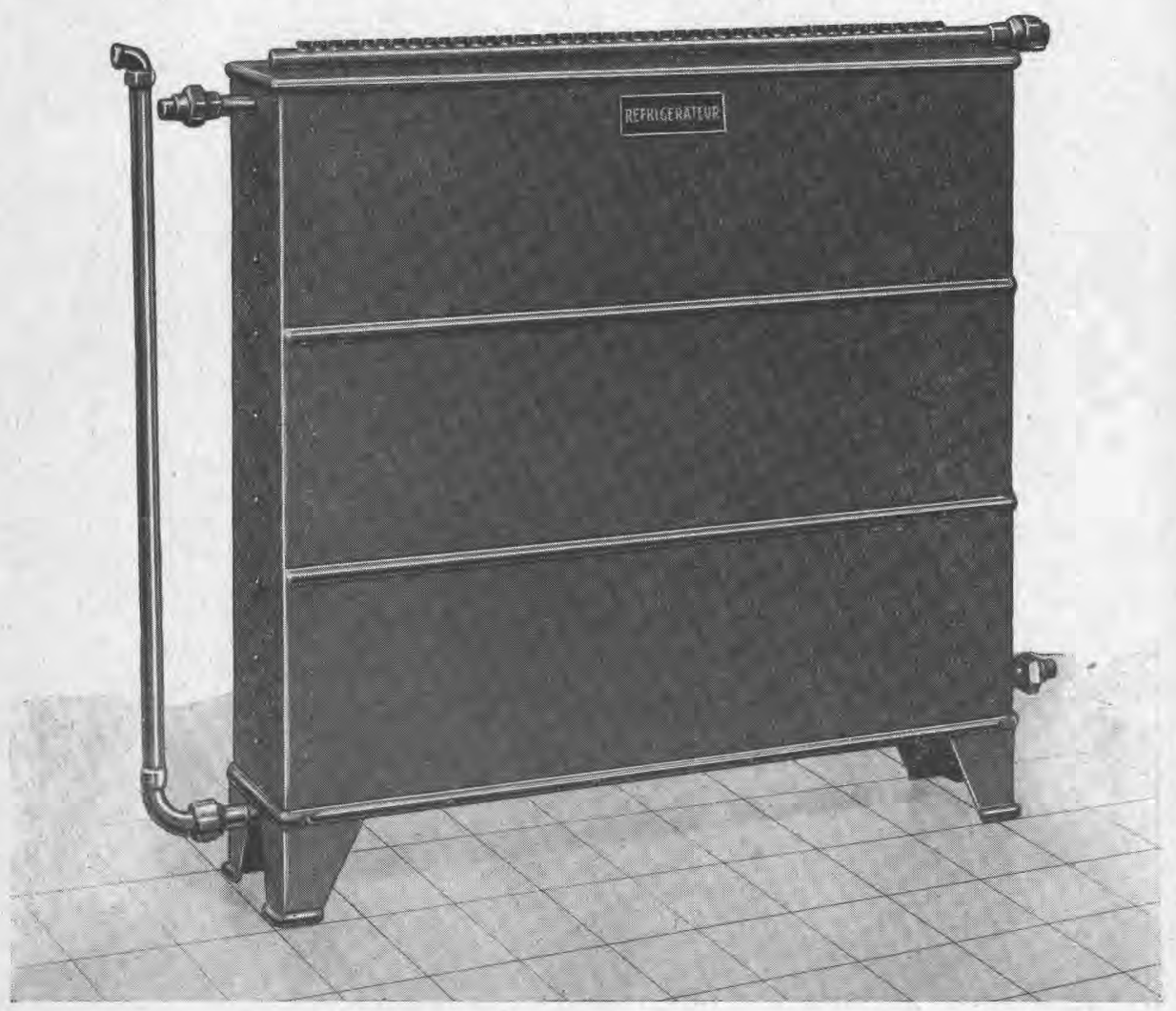

Réfrigérant, système W. P. de Stoutz sur circuit fermé, débit 1.800 litres heure.

première fois la formule de l'écoulement laminaire des fluides pour leur irradiation. Ses recherches lui ont permis de déterminer un régime nouveau d'écoulement permettant un débit considérable et assurant l'actinisation des fluides dans toute leur masse.

Tous ces détails montrent comment cet appareil a été conçu, pour les plus petits effets de ses moindres détails.

\section{Actinisateur W. P. de Stoutz. - Son utilisation en laiterie.}

Une fois l'appareil réglé pour une intensité d'irradiation donnée,

(1) L. Crussard. Grandes Encyelopédies Industrielles. J.-B. Baillière, page 60 , ch. VIII. 
il n'est plus nécessaire de l'ouvrir. Il est même recommandé de le tenir fermé à clé pour qu'on ne vienne pas d'une façon inconsidérée toucher aux lampes ou à la harpe.

Le lait arrive par la partie inférieure, remonte par les tubes capillaires où il est irradié et s'écoule ensuite dans un réfrigérant

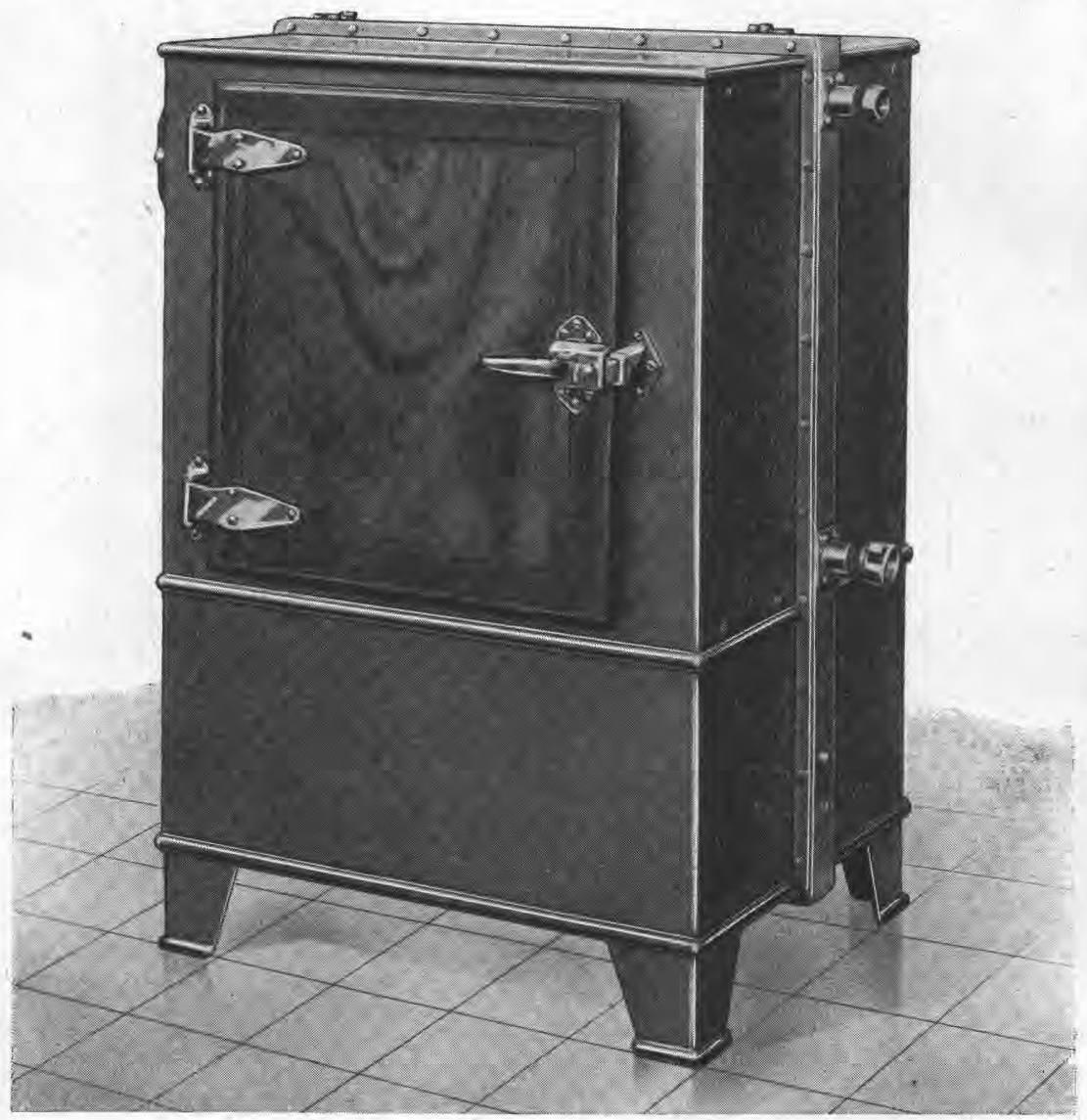

Actinisateur, côté entrée et sortie du lait.

par le collecteur supérieur pour être ensuite mis en bouteilles, bidons ou réservoirs de garde. Le débit est de 1.500 litres-heure et la quantité totale se trouvant dans les tubes capillaires est d'environ $40 \mathrm{~cm}^{3}$. On peut n'irradier que quelques litres à la fois, s'arrêter quand on le veut, en fermant le robinet de sortie, le lait ne chemine plus, et malgré que les lampes soient toujours allumées, l'irradiation, étant donné l'opacité du lait, ne se fait pratiquement plus. En effet, pour que l'irradiation ait lieu, il est indispensable que le fluide 
soit en mouvement dans l'appareil. Il serait d'ailleurs facile d'éteindre synchroniquement les lampes lors des arrêts du courant de lait.

Lors de la recherche de la meilleure intensité pour irradier du lait, on est arrivé à actiniser 23 échantillons de 10 litres chacun, en moins de 3 heures, l'appareil étant nettoyé par l'eau stérilisée aux rayons ultra-violets et par la vapeur d'eau également stérilisée entre chaque opération, il y eut prise d'échantillon et changement d'intensité.

Les industriels laitiers, qui savent le temps que doivent passer leurs employés pour nettoyer les appareils existants d'une façon à peu près satisfaisante, comprendront aisément l'importance de ce maniement : 9 minutes pour irradier différemment 10 litres de lait, prendre des échantillons et nettoyer aseptiquement tout l'appareil.

Pour le nettoyage, un jeu de vanne ; les lampes à rayons ultraviolets fonctionnant, on fait pénétrer de l'eau facilement, car le lait froid s'enlève sans peine; une purge de l'eau et l'on fait pénétrer la vapeur d'eau; celle-ci permet d'enlever le calcaire que l'eau aurait pu déposer et l'on est ainsi assuré d'avoir des tubes constamment perméables aux rayons ultra-violets.

Avec la pasteurisation calorique, où il fallait tenir compte du peu de conductibilité du lait, la couche mince était de l'ordre de $1 \mathrm{~mm}$., l'action des rayons se faisant sentir environ 30 secondes ; avec les rayons chimiques, la couche mince est de $1 / 100^{\mathrm{e}}$ de millimètre et le temps de $1 / 1.000^{\mathrm{e}}$ de seconde. StasSANo était arrivé pour des cultures microbiennes à $1 / 100^{\mathrm{e}}$ de millimètre pendant une ou deux secondes. Avec le "Stassano", ce qui étrit difficile, e'était de transformer un appareil de laboratoire en une machine industrielle (Coepfert, page 49).

Avec l'Actinisateur W. P. de Stoutz, on arrive du premier coup à l'appareil industriel qui peut servir d'appareil de laboratoire. Il n'y a pas de modifications à y apporter.

\section{CONSIDÉRATIONS SCIENTIFIQUES ET RECHERCHES POSSIBLES}

\section{AVEC L'ACTINISATEUR}

Les rayons ultra-violets et l'actinisation du lait nous ont amenés aux considérations suivantes concernant les diverses branches de l'activité scientifique, considérations qui peuvent amener des recherches nombreuses et possibles grâce à l'actinisateur de Stoutz. Chimères, peut-être, mais il suffit d'une anticipation probable pour que les chercheurs s'engagent dans cette voie nouvelle, où nous leur souhaitons une ample moisson. Pour nous, les résultats obtenus en laiterie sont tels que nous poursuivons les recherches pour nous les expliquer et les exploiter.

Médecine, - En médecine humaine comme en médecine vété- 
rinaire, des essais nombreux, depuis Finsen, ont été tentés avec les rayons eux-mêmes, et depuis Stenbock et Daniels, avec les produits irradiés.

Mais l'irradiation avait lieu sur la surface des corps opaques, donc elle ne pouvait être que très faible et très inégale. Si l'on a eu des résultats appréciables de cette manière, ceux obtenus avec des produits irradiés en couche mince n'en seront que plus grands et plus certains. Evidemment une technique de l'actinisation devra être cherchée pour chaque produit, comme nous la cherchons actuellement pour les branches de la laiterie. En tous les cas l'irradiation en un temps total moindre de $1 / 10^{\mathrm{e}}$ de seconde ne pourra avoir les inconvénients rencontrés souvent avec les irradiations de plusieurs secondes. M. le Dr Lesné reconnaît dans lo No 153 de $\mathrm{I}^{\text {' }}$ "Hygiène sociale " :

“ $1^{\circ}$ Que la vitamine D est renfermée dans peu d'aliments. Qu'elle existe dans les oufs et le lait, mais que pour ces aliments elle varie avec l'alimentation et le genre de vie des animaux : vaches et poules vivent-elles en plein air, exposées aux rayons solaires, la vitamine D augmente dans le lait et l'œuf ; elle est plus abondante dans le lait d'été que dans le lait d'hiver.

$2^{\circ} \mathrm{Qu}$ 'en faisant ingérer soit à des nourrices soit à des vaches laitières une huile de foie de morue riche en facteur $D$, cette vitamine ingérée est éliminée par le lait qui devient antirachitique.

$3^{\circ}$ Que l'exposition du lait aux rayons ultra-violets doit se faire à l'abri de l'air.

$4^{\circ}$ Que jusqu'à présent les périodes d'irradiation allaient de quelques secondes jusqu'à 2 heures.

$5^{\circ}$ Que d'après les expériences de SUPPLEE et DorCas il semblait que le pouvoir antirachitique maximum susceptible d'être donné au lait l'était en général dans les toutes premières secondes d'exposition.

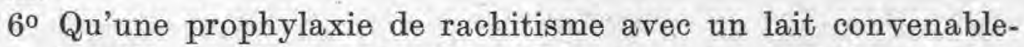
ment irradié est évidemment possible pour un eertain nombre d'auteurs, mais l'exécution pratique en est difficilement réalisable.

$7^{\circ}$ Le lait irradié vendu dans le commerce est sec ou condensé, il est biologiquement modifié et son emploi supprime donc le lait frais.

C'est un mode d'alimentation de prix élevé. "

Personnellement, nous croyons qu'un lait frais provenant de vaches saines, recueilli proprement, exposé aux rayons ultra-violets pendant moins de $1 / 10^{\mathrm{e}}$ de seconde avec une intensité minimum dans l'appareil W. P. de Stoutz et conservé au frais, en un mot du lait que nous qualifions d'actinisé, doit répondre aux exigences des pédiatres les plus rigoureux. Au lacto-fermentateur il donne un 
eaillé analogue au meilleur lait de printemps. Des expériences sur des enfants de la Crèche Municipale de Grenoble ont donné, par comparaison, des effets très encourageants et pas un effet nocif.

Grâce aux ferments lactiques toujours vivants et plus virulents à la température du corps, le lait s'acidifie plus rapidement et est de ce fait d'une digestibilité plus grande. Ce lait est plus vivant que n'importe quel lait, mais avec une flore bactérienne améliorée. Cette qualité peut avoir en même temps un léger inconvénient, car peu de mères de famille comprennent que le lait doit être maintenu au frais (au-dessous de $12^{\circ}$ ) jusqu'au moment de son emploi, car à $25^{\circ}$, à $30^{\circ}$, il caillera plus facilement qu'un lait pasteurisé.

Evidemment, pour supprimer cette "qualité-défaut ", il sera loisible à l'industriel laitier de pasteuriser comme un lait ordinaire le lait actinisé, la vitamine D persistera, la facilité d'acidification disparaîtra, la ménagère sera contente, le pédiatre le sera moins.

Hydraulique. - On est surpris du débit qu'a l'appareil W. P. de Stoutz dont la somme des surfaces de section des tubes capillaires est moindre que la section des collecteurs d'arrivée et de départ. La vitesse est accrue malgré les résistances aux frottements et à la pesanteur. Cette observation pourrait donner aux spéléologues certaines explications, quant au débit des cours d'eau souterrains, et confirmerait certaines hypothèses sur les écoulements laminaires.

La vitesse du lait, liquide légèrement visqueux, est dans les tubes capillaires de l'ordre de 5 mètres seconde; on comprend done qu'à cause de la résistance due à la viscosité, il y ait un étalement laminaire et que toutes les parties de la goutte de lait soient projetées tour à tour contre les parois pour une irradiation parfaite en couche mince. Il y a un étirement de la goutte avec épanouissement à la partie supérieure.

Il serait intéressant de pouvoir faire passer dans l'appareil un liquide tenant en suspension de fines particules coloriées ou phosphorescentes, liquides ayant la même viscosité que le lait pour le cinématographier au passage et projeter ensuite le film au ralenti afin de bien se rendre compte du phénomène hydraulique.

Actinologie. - Il faudrait rechercher, grâce au spectre, la partie active pour chaque processus biologique que l'on peut considérer dans l'irradiation du lait, afin de conserver les bons et éliminer les mauvais.

Lorsqu'on compare, sur le schéma de SaIdman, les différences de longueur d'onde, infra-rouge et ultra-violet, par rapport aux dimensions d'un globule rouge, d'un bacille de Koch ou d'un staphylocoque d'après un grossissement apparent égal à 10.000 , on se rend compte qu'il faut une forte émission d'infra-rouge pour arriver à 
impressionner la partie vivante de ces différents organismes et y apporter les troubles chimiques.

La proportion des longueurs d'onde $\frac{\text { infra-rouge }}{\text { ultra-violets }}$ paraît être du même ordre de grandeur que la proportion $\frac{\text { radioélectrique }}{\text { infra-rouge }}$. Or, les radioélectriques, qui sillonnent l'espace actuellement avec la T. S. F. et viennent nous bombarder d'une façon presque continue, le font sans dégagement appréciable de chaleur.

On peut done conclure que le pouvoir microbicide de la chaleur est dû d'abord à une action sur le milieu lui-même, comme cela paraît se passer pour les rayons ultra-violets.

Chimie-Photochimie-Histochimie. - Que se passe-t-il dans le lait sous l'influence de l'actinisation? Quels sont les éléments qui sont touchés et dans quelles proportions ? Des expériences multiples sont à faire, et on peut les répéter pour chaque liquide.

Les effets sont fonction de l'intensité et de la durée de l'irradiation. Les différences observées sur les laits de même provenance le prouvent. Ils sont aussi fonction de l'état dans lequel se trouve le lait au départ.

Nous avons donc 3 variables qu'il faut connaître avant d'irradier pour essayer d'obtenir, à la fin, les résultats recherchés.

La diminution de la densité et les essais au lacto-fermentateur sont les preuves de ces différences.

Des essais chimiques nombreux sont à faire pour constater, sur les éléments constitutifs du lait, l'action des rayons ultra-violets et essayer d'en expliquer le mécanisme.

L'appareil W. P. de Stoutz peut devenir précieux pour toutes ces recherches, qui permettent de mieux comprendre l'influence du soleil par une partie de ses radiations, dans les phénomènes biochimiques, les oxydations, réductions, condensations, polymérisations, etc., sont les réactions complexes de la photochimie. Par la photochimie nous connaîtrons ainsi les phénomènes qui se passent dans la partie morte ou non vivante du lait, avec l'histochimie nous pénétrerons dans le coeur de la vie.

Influence des rayons ultra-violets sur la matière vivante! Nous pouvons mieux nous rendre compte des échanges nutritifs entre les milieux et les êtres vivants : microbes-cellules végétales ou animales et de l'influence microbicide de ces radiations.

Par l'étude de ces influences nous arriverons à doser les rayons ultra-violets pour avoir l'effet optimum.

Microbiologie. - Des expériences nombreuses sont à faire en cette matière. 
Les rayons ultra-violets peuvent agir histologiquement sur les microbes eux-mêmes, mais ceux-ci protégés partiellement par une enveloppe moins perméable il faudrait des intensités plus fortes. Ceci demande encore à être contrôlé.

Pour les microbes pathogènes ou saprophytes du lait il serait du plus haut intérêt de faire deux séries de cultures : l'une dans des tubes ordinaires, l'autre dans des tubes de quartz, d'irradier ces derniers, et de faire l'examen comparatif microscopique et d'essayer ensuite les vitalités respectives soit in vitro pour de nouvelles cultures, soit in vivo par inoculation à des animaux sensibles.

On se rendrait compte si les microbes vus au microscope sont encore vivants, ou ne sont plus que des cadavres n'agissant plus que par leurs toxines, s'ils en avaient émis.

On pourrait aussi se rendre compte plus parfaitement de l'influence mierobicide par action sur le milieu en irradiant ceux-ci avant ensemencement. Si un résultat positif était obtenu, on pourrait se rendre compte de la vitesse de l'effet microbicide du milieu. S'il n'était que lent, on ne pourrait pas utiliser un tel procédé pour application thérapeutique.

Avec l'appareil W. P. de Stoutz, il serait facile de faire des injections avec des liquides fraîchement irradiés.

Bromatologie. - En France, à l'heure actuelle, lorsqu'une ménagère achète son lait quotidien, elle commence par le faire bouillir, fût-il cru, pasteurisé, stassanisé.

En France, peu de ménages, -malheureusement, ont à leur disposition le froid. Mais, souvent, dans certaines villes, et à Grenoble en particulier, on a l'eau fraîche à $9^{\circ}$ à volonté et cela pendant toute l'année. Dans ces villes on peut done consommer facilement le lait pasteurisé ou stassanisé sans le faire bouillir. On le ramène à $35^{\circ}$ au moment de son ingestion pour les jeunes organismes. Il est facile de construire un appareil ménager pour une ou deux bouteilles de lait capable de tenir au frais, par un léger courant ascendant d'eau fraîche.

Pour le lait actinisé, c'est le procédé de choix à recommander. Ne pas détruire par un ehauffage intempestif les qualités que l'irradiation lui a données.

Le lait actinisé reste vivant, aussi vivant que n'importe quel lait cru, par l'effet sélectif des rayons ultra-violets sur les milieux nutritifs ; les bacilles lactiques cultivent plus facilement que les autres, augmentant ainsi la digestibilité du lait.

Les effets nuisibles signalés par de nombreux auteurs, par l'irradiation de longue durée, ne peuvent être retenus ici, puisque le passage dans les tubes capillaires se fait en $1 / 10^{\mathrm{e}}$ de seconde et que 
Supplee et ses collaborateurs ont montré qu'une exposition de quelques secondes n'a aucun effet fâcheux.

Le pouvoir antirachitique est toujours considérablement augmenté, il est même créé de toutes pièces, en l'absence de la vitamine D, par la transformation de l'ergostérol.

Le lait de provenance contrôlée, au point de vue bactériologique soit à l'étable, soit à la laiterie, est donc, après actinisation, le plus recommandable non seulement aux nourrissons, mais aussi aux mères allaitant et à tous ceux qui ont à lutter contre le rachitisme sous toutes ses formes. C'est donc affirmer qu'il devrait être d'un emploi général dans l'alimentation.

Physiologie. - Des analyses nombreuses ont montré les différences quantitatives et qualitatives suivant les espèces, les saisons, la nourriture, etc.

Notre collègue et ami, le $\mathrm{D}^{\mathrm{r}}$ Marcel Couturier, nous a apporté dernièrement un renseignement précieux sur la teneur en matière grasse du lait de chamois.

Ces différences chez les espèces conditionnent la vie des jeunes.

L'homme actuel vit-il dès sa naissance une vie normale? Sa mère est-elle capable de lui donner pendant les premiers mois la nourriture la plus apte à sa croissance et à sa vitalité ?

A notre connaissance on n'a pas encore fait d'étude comparative sur les propriétés antirachitiques des laits des différentes espèces suivant les latitudes et l'intensité solaire, et chez la femme l'influence des vêtements et de l'héliothérapie.

Influences quantitatives et qualitatives, tout est sujet à recherches. Connaissant ainsi par comparaison les besoins, il sera facile d'y remédier chez les animaux supérieurs lors d'une carence par un lait convenablement actinisé.

"Chaque enfant coûte une dent à sa mère", dit-on souvent. Il serait du plus haut intérêt de se rendre compte, si les nourrices consommant du lait actinisé seraient moins sensibles que les autres et si leur denture serait plus résistante. Il faudrait aussi se rendre compte de la dentition de leur progéniture. Expérience longue peutêtre ; mais en biologie humaine ce n'est qu'après un long terme que l'on peut se rendre compte de certains résultats.

Lorsqu'on compare les dentitions actuelles avec celles de nos aîeux, au temps où la prothèse n'existait pas, on est en droit de penser que notre vie actuelle, notre nourriture sophistiquée, font partie des causes de cette déficience facile à contrebalancer.

Désinfection. - Où le soleil entre, la maladie ne pénètre pas. Avec les rayons ultra-violets on peut remplacer non le soleil en entier, mais les radiations de celui-ci, qui ont un pouvoir microbicide puissant. 
Leur emploi dans la désinfection serait, à notre avis, plus facile que les méthodes utilisées jusqu'à ce jour.

Comme vétérinaire, j'estime que pour la désinfection des étables, il y aurait là un procédé de choix.

On pourrait aussi envisager l'emploi des rayons ultra-violets pour le conditionnement des salles d'opérations et des chambres de malades.

D'autres branches de l'activité humaine : agriculture, botanique, par eux, pourraient trouver à notre avis un terrain propice aux investigations fructueuses.

\section{L'ACTINISATION ET LA PRATIQUE LAITIÈRE}

Il faut reconnaître que les essais faits jusqu'à ce jour sur l'actinisation des laits, l'ont été plus au profit des recherches de laboratoire et de l'étude du rachitisme qu'à celui de la pratique laitière avec une matière première dont la qualité ne justifiait pas toujours les efforts entrepris.

Le lait actinisé est à l'heure actuelle un produit pharmaceutique destiné à lutter contre le rachitisme, quoique beaucoup de médecins le déconseillent parce que, d'après eux, il n'a d'autre garantie que celle du fabricant et est de ce fait un moyen thérapeutique aveugle. Ils n'ont pas toujours tort, et pourtant sur ce sujet nous avions les essais concluants de Kramer, Madkayr, SHaw, Gyorgy.

C'est à notre sens trop restreindre le sujet que de ne le considérer qu'au point de vue antirachitique. Il y a encore autre chose : pour nous le lait actinisé est et doit être l'aliment par excellence de l'enfant et surtout du nourrisson privé du sein maternel, Car, en plus de son enrichissement en pouvoir antirachitique, il est devenu plus sain, parce que plus pauvre en microbes, bien que le développement de la flore lactique soit nettement favorisé ; plus savoureux, plus assimilable, en un mot plus près de son état naturel, ainsi que nous l'avons découvert quand, au cours des premiers essais, nous nous sommes aperçus que les caillots des lacto-fermentateurs des laits actinisés étaient toujours d'une qualité supérieure, et parfois complètement différente des caillots des laits crus ou pasteurisés.

Il est vrai que l'appareillage et la technique de l'actinisation étaient jusqu'à ces derniers jours à peu de chose près ce qu'ils étaient lors de sa découverte et de la première machine créée par SEIFFERT, à Hamburg, en 1903. Seule la couche mince entre deux lames de verres spéciaux ayant succédé au ruissellement sous pression de $\mathrm{CO}^{2}$. Le goût d'huile de foie de morue n'avait jamais été complètement supprimé, bien au contraire certains laits irradiés étaient imbuvables, et le débit ne s'élevait jamais qu'à quelques cent litresheure. Aussi, en tenant compte de ces deux points, des difficultés 
de nettoyage et de conduite des appareils nécessitant souvent la présence d'un technicien, l'industriel le moins averti s'apercevait que "ça ne rendait pas »; ceei est aujourd'hui une question primordiale pour l'industrie laitière, laquelle n'a rien à voir avec la philanthropie. Il y a bien des essais faits par Supplee et Dordas, de la "Dry-Milk Co.", de New-York (voir "Le Lait", janvier et février 1934), sur des quantités dites industrielles (450 litres), mais les détails manquent un peu sur la technique d'irradiation, et le temps employé.

Avec l'appareil de Stoutz, la question du débit et de toute absence de goût étant définitivement réglée, on doit s'attendre à ce que l'actinisation fasse de grands progrès non seulement dans la pratique laitière, mais aussi quant au sujet des recherches sur les transformations physiques et chimiques du lait actinisé. Car jusqu'à maintenant le seul sujet étudié, et pourtant objet de tant de controverses, est celui du rachitisme. Sans mésestimer ce point, qui mérite l'importance qu'on lui attribue, on ne peut que regretter la pauvreté, sinon l'absence de toute recherche, sur le lait actinisé même. Ne serait-ce, que comme complément de toutes ces méthodes rationnelles de traitement industriel du lait dans les laiteries et surtout de la pasteurisation par les transformations qualitatives que l'actinisation apporte au lait. La pasteurisation a complètement perdu son sens primitif. L'intensité de la produetion, l'affouragement irrationnel au moyen des toúrteaux, des pulpes ou des drèches, l'emploi parfois irraisonné des engrais chimiques, les conditions déplorables de ramassage du lait dans la plupart des régions, etc., tout cela a forcé les industriels laitiers à considérer la pasteurisation moins comme un moyen d'obtenir un lait hygiénique et d'augmenter sa conservation que comme le seul moyen à leur portée pour parer aux caprices de l'acidité, et de leur permettre ainsi de livrer aux consommateurs un lait qui ne "tourne " pas à la cuisson après 24 ou 36 heures. Nous en voyons la preuve dans ees puissantes organisations qui de la production à la vente se voyaient dans l'obligation de pasteuriser plusieurs fois leur lait.

D'autre part, quand on a été tant soit peu en contact avec la clientèle de détail, quel chapitre ne ferait-on pas sur les notions plus que rudimentaires de la majorité des ménagères et, partant, des mères de famille quant au lait. Nous croyons qu'il ne faut pas chercher ailleurs la cause de cet empressement à employer tous ces laits qui n'en ont que le nom, pour l'alimentation des nourrissons, dans des pays où le lait est produit en abondance et pourrait avec un peu de bonne volonté être produit sain.

La pasteurisation a été une belle réalisation pratique en industrie laitière, mais hélas, depuis quelques années, elle en est devenue le lit de paresse. Quand nous voyons tous les efforts que l'on tente 
actuellement pour améliorer les traitements du lait dans les laiteries, nous pensons toujours à cette ville qui aurait fait installer une prise d'eau à côté d'une bouche d'égout et qui se préoccuperait davantage de filtrer son eau par tous les moyens que de changer sa prise d'eau de place.

Nous croyons qu'il faudrait s'occuper un peu moins de tous ees traitements mécaniques et un peu plus de la production. Car sinon nous arriverons bien un jour à fabriquer du lait synthétique, beaucoup crieront au miracle et nous annonceront le régime des. plus beaux enfants, de tous les temps. Il est vrai aussi qu'avec un peu d'adroite publicité !...

Le ramassage du lait de ferme en ferme et aux abords des chemins et des haies a fait son temps. C'est cela qu'il faut supprimer. Une seule solution, le local de coulage par village où soir et matin les producteurs apporteront leur lait. Les régions où se fabriquent les pâtes dures s'en trouvent bien, et n'adopteraient certainement. pas d'autre façon de faire. Et quelle facilité pour le contrôle.

(A suivre.)

\title{
L'APPROVISIONNEMENT EN LAIT DE LA NATION. PRODUCTION HYGIÉNIQUE ET CONTROLE
}

\author{
par BEN DAVIES
}

Directeur des laboratoires "United Dairies Limited" (Londres).

$$
\text { (Fin) }
$$

Un intéressant corollaire de cette expérience fut fourni lorsque six des douze fermiers, après leur expérience de production de lait propre, furent invités à visiter six autres producteurs et à faire la traite de leurs vaches, un après-midi, pour démontrer ainsi quelle amélioration pouvait être obtenue, sans aucune préparation spéciale, mais en se servant de leurs propres ustensiles stérilisés, qu'ils prirent avec eux. La première colonne du tableau suivant donne les.

TABLEAU E.

\begin{tabular}{|c|c|c|c|}
\hline \multirow{2}{*}{\multicolumn{2}{|c|}{ Ferme }} & Propriétaire & Visiteur \\
\hline & & $6 / 3 / 33$ & $10 / 3 / 33$ \\
\hline 1 & $\ldots \ldots \ldots \ldots$ & 3.000 .000 & 2.500 \\
\hline 2 & $\ldots \ldots \ldots \ldots$ & 3.000 .000 & 1.900 \\
\hline 3 & $\ldots \ldots \ldots \ldots$ & 25.400 & 5.500 \\
\hline 4 & $\ldots \ldots \ldots \ldots$ & 70.000 & 2.900 \\
\hline 5 & $\ldots \ldots \ldots \ldots \ldots$ & 24.500 & 900 \\
\hline 6 & $\ldots \ldots \ldots \ldots$ & 3.000 .000 & 500 \\
\hline
\end{tabular}

